\title{
Nutritional labelling of takeaway food: scope for physical activity equivalents?
}

\author{
T.M. Blackham ${ }^{1}$, A. Hands ${ }^{2,3}$, P. Hargrave ${ }^{3}$, I.G. Davies ${ }^{1}$, L. Stevenson ${ }^{1}$, \\ V. $\operatorname{Cox}^{2}$ and A. Coufopoulos \\ ${ }^{1}$ Food, Nutrition and Health Research Group, Liverpool John Moores University, L17 6BD, ${ }^{2}$ Coventry University, \\ CV1 $5 F B$ and ${ }^{3}$ Coventry City Council, CV1 5RS.
}

Takeaway food consumption has been associated with obesity and related diseases ${ }^{(1)}$ and the UK Government are campaigning to reduce population level calorie intake ${ }^{(2)}$. Nutritional information is frequently provided by larger fast food chains; however, this information is less commonly provided by small independent takeaways. Previous research has demonstrated the effectiveness of physical activity labels in reducing energy at point of purchase ${ }^{(3)}$. The present study aims to compare the energy value of takeaway food using the front-of-pack "traffic light" scheme, the calories only system, and "physical activity equivalents" or exercise labels. Total energy was converted to jogging hours using metabolic equivalent (METS) intensity values ${ }^{(4)}$.

A public engagement exercise was carried out at a food and drink festival in Liverpool to assess frequency of takeaway food consumption and opinions about nutritional labelling. Posters of popular takeaway food were displayed together with examples of calories only, traffic lights and physical activity equivalent labels. Members of the public were asked to study the posters and complete a short takeaway questionnaire. Data collection was carried out over two days at the festival and analysed using descriptive statistics.

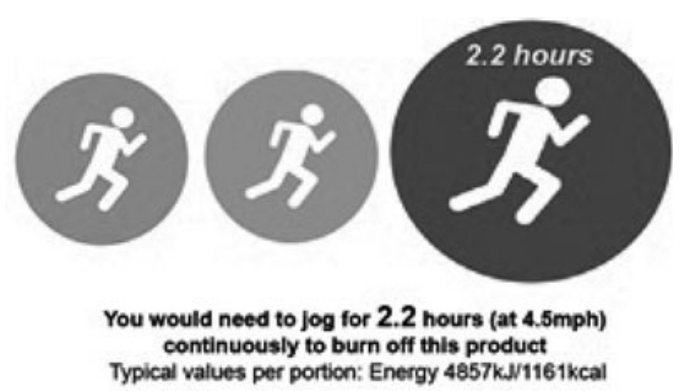

Fig. 1. Example Physical Activity Equivalent Label

In total 172 adults (aged 18+) completed the questionnaire (59 men, 111 women, 2 unspecified). Only two respondents did not eat takeaway food; $29 \%$ of respondents ate takeaway food 1-2 times a week compared with $47 \%$ who ate it 1-2 times a month. A large proportion of respondents $(83 \%)$ felt that nutritional labelling on takeaway food was a good idea, with calories $(70 \%)$ and fat $(64 \%)$ being considered the most useful. The majority of respondents $(94 \%)$ were interested in purchasing healthier takeaways, particularly meals containing less fat $(69 \%)$ and calories $(59 \%)$. When asked which of the three choices of nutritional labelling was easier to understand, $66 \%$ of respondents chose traffic light labelling, $18 \%$ physical activity labelling and $11 \%$ calories only. A few respondents $(5 \%)$ suggested that a combination of traffic light and physical activity equivalent labels would be useful.

In conclusion, this study offers an alternative method of presenting calorie information on takeaway food. Although a large proportion of respondents chose traffic lights, 30 respondents preferred physical activity equivalents and 8 respondents felt that a combination of the two would be helpful. Previous studies have shown physical activity equivalent labelling to be effective in the USA ${ }^{(5)}$. Further research on the various formats of nutritional labelling of takeaway food is therefore warranted to reduce energy intake from foods purchased from the fast food and takeaway industry, particularly when the energy content of many takeaway foods provided by small independent takeaways is high $^{(6)}$.

1. Jaworowska A, Blackham T, Davies IG, et al. (2013) Nutr Rev 71(5), 310-18.

2. Public Health England (2018) Calorie reduction: the scope and ambition for action. March 2018. https://www.gov.uk/government/publications/ calorie-reduction-the-scope-and-ambition-for-action.

3. Bleich SN, Herring BJ, Flagg DD et al. (2013) Am J Public Health 104 (12), 2417-24.

4. Ainsworth BE, Haskell WL, Whitt MC et al. (2000) Med Sci Sports Exerc 32(9 Suppl), S498-S504.

5. James A, Adams-Huet B \& Shah M (2015) Am J Health Promot 29(5), 294-302.

6. Jaworowska A, Blackham T, Long R et al. (2014) Nutr Food Sci 44(5), 414-30. 